\section{Difference between conventional and modern methods for examination of fingerprints}

\author{
Ambati Ramesh Babu* \\ Lecturer, Forensic Science, Aditya Degree College, Surampalem, Andhra Pradesh, India
}

\section{Abstract}

The impression of frictional ridges of the finger is known as fingerprints. Owing to this uniqueness, the fingerprints have long been used to identify a person since Ancient times. In any crime scene the presence of fingerprint makes the identification of the Culprit very easy. The fingerprints can also easily be embedded on any item such as paper, Clothing and body of the victim. To utilize this uniqueness of fingerprints forensic experts devised many techniques to obtain a clear fingerprint. These come under two categories i.e. Conventional and modern methods.

The conventional methods are although important but there are limitations of them. Just take the example of powder method. Powder method require different powders for different Surfaces and colors, but modern method like quantum dots method can easily detect Fingerprints on all surfaces regardless of their color giving great resolution in seconds. Other methods like physical developer method is very time consuming and expensive, carbon Black method creates mess and does not work on porous surface, iodine fuming and Naphthaloflavin does have an advantage that it can bring up prints on skin also but it does not Work on metallic surfaces. VMD also fails on heavy plastic polymers and body oils. But some modern methods like nanotechnology can obtain high resolution prints old and dried prints also within 3 minutes. Laser technology is very fast, accurate and can be used for Fingerprints up toten years old also on any surface without any mess. Multimetal deposition Method can even be used to identify smokers and drug addicts and can be used Porous, non-porous and wet surfaces.

\section{More Information}

*Address for Correspondence: Ambati Ramesh Babu, Lecturer, Forensic Science, Aditya Degree College, Surampalem, Andhra Pradesh, India, Tel: 7382897399; Email: rameshbabuambati96@gmail.com

Submitted: July 20, 2021

Approved: August 09, 2021 Published: August 10, 2021

How to cite this article: Babu AR. Difference between conventional and modern methods for examination of fingerprints. J Forensic Sci Res. 2021; 5: 037-040

\section{DOI: 10.29328/journal.jfsr.1001025}

Copyright: ๑ 2021 Babu AR. This is an open access article distributed under the Creative Commons Attribution License, which permits unrestricted use, distribution, and reproduction in any medium, provided the original work is properly cited.

Keywords: Laser: Light Amplified Stimulated Emission Radiation; QD: Quantum Dots; MMD Multi Metal Deposition; VMD: Vacuum Metal Deposition

Check for updates

OPEn ACCESS

\section{Introduction}

Fingerprints are gods own seal that are given to us that we may recognize his best creation-man.

\section{Pattern classification}

All finger prints can be divided into four basic types:

ARCHES, LOOPS, WHORLS AND COMPOSITES. Again are further subdivided in to the following types:

\begin{tabular}{|c|c|c|}
\hline Basic Pattern & Sub Division & Symbol \\
\hline 1. Arches & Plain arch Tented arch & A T \\
\hline 2. Loops & Radial & R \\
& Ulnar & U \\
\hline 3. Whorls & & $\mathrm{W}$ \\
\hline & Lateral pocket loop & LP \\
\hline \multirow{2}{*}{ 4. Composites } & Central pocket loop & $\mathrm{CP}$ \\
& Twin loop & $\mathrm{TL}$ \\
& Accidentals & $\mathrm{AC}$ \\
\hline
\end{tabular}

\section{Finger print developing kit}

When visiting a scene of crime, finger print experts carry with them a latent print development kit consistence of variety of powders and brushes, lifting materials, magnifying glass, flash lights, two pairs of gloves, one pair of scissors, one pocket knife, one tape, fingerprinting kits and slips.

\section{Technique preference}

The technique to the used for the development of latent fingerprints depends on various factors:

1) Nature of crime

2) Time of happening

3) Nature of surfaces rough or smooth surfaces, wet or dry surfaces, surface contamination blood or grease etc

4) Size of article. 


\section{Developing a scene of crime fingerprints}

Visual examination: crime scene is checked for latent fingerprints by using oblique light sources. Sometimes latent fingerprints are revealed if the surface is clean and smooth.

Fluorescence examination: High intensity light sources using different filters are also useful to reveal fingerprints.

Technique preference: The technique to the used for the development of latent fingerprints depend on various factors

1) Nature of crime

2) Time of happening

3) Nature of surfaces rough or smooth surfaces, wet or dry surfaces, surface contamination blood or grease etc

\section{Coventional methods of fingerprint development}

Powder development techniques: If the powdering techniques referred, it is not important to determine which powder to be used the powder selected should be fine grained and of a color that gives contrast with the background.

On dark surfaces grey or white powder is used whereas on light surfaces black powder is used. Original single base powders like lampblack or finely ground charcoal was used on light surfaces and lead powder or cigar ash was found useful on dark surfaces. But this powder has come to be known in the recent past making use of more than one ingredient.

Black powder: Results are obtained with this powder when applied on paper and chinaware.

Grey or white powder: Excellent obtain from glassware, silver wares and pelted wares.

Red powder: Useful to develop fingerprints on paper where the prints are fresh.

Silver powder: It is used hard surfaces which are painted, polished or vanished and objects like cellophane.

Physical developer: Used for developing prints on wet surfaces because organic residues are insoluble in water so the physical developer can detect latent impression.

- Produce dark grey or black impression.

- Used porous surfaces

- Used when nihydrin and iodine fails to work.

\section{Disadvantages:}

- Time consuming Expensive

- $\quad$ Destructive

- $\quad$ Short Shelf Life

\section{lodine fuming}

Principle: Natural body fats and oils sebaceous material temporarily absorb the iodine vapor. This result in a change in color from clear to a dark brown until the effect fades with time IODINE FUMING is used to reveal prints on porous and semi porous surfaces such as paper, cardboard, and unfinished wood but not on metallic surfaces

- One of the earliest methods $\bullet$ Inexpensive

- $\quad$ Easy

\section{Carbon black:}

- Consists of charcoal and binder.

- Applied on light color surfaces even show up on black glossy surfaces as light colored.

- Produce dark gray image

Disadvantages:

- $\quad$ Create mess

- Cannot work on porous surface

\section{Ninhydrin:}

- $\quad$ First used in 1959

- Ninhydrin used on porous surfaces like paper and wall

- It reacts with free amines of lysine residue in protein sloughed off in fingerprints Produce deep purple color

- Applied by spraying or dipped. Development is done in highly humid environment because water is necessary for reaction

- Stored in dark place to avoid degradation

Disadvantages:

- Development need long time without humidity

- Storage is difficult because it is degraded after some time

Specimens that have been successfully treated using VMD

- The questioned surface is placed in a chamber from which the air is evacuated

- The chamber also contains small pieces of gold and zinc that can be heated electrically to vaporize

- Zinc cannot deposit on the oily residues present in the fingerprint but gold can deposit on the fingerprint but gold can deposit on the entire surface. Gold is absorbed into oil containing ridges of the fingerprint thus there will be no gold on the surface of the ridges 


\section{Disadvantages:}

- Effectiveness can be reduced by the presence of body fluids and drugs residues

- Difficult to develop prints on heavily plasticized polymer

- Requires expensive equipment and material

\section{Modern methods of fingerprint development}

Quantum dots: In the last few years, another approach that has garnered a lot of attention is the use of quantum dots (QDs) for fingerprint detection. QDs are interesting because they fluoresce strongly under UV light and have varying emission spectra based on their particle size. The type of QDs most studied in the context of fingerprint detection are ones that derive from cadmium, namely cadmium sulphide (CdS), cadmium selenide ( $\mathrm{CdSe}$ ), and cadmium telluride ( $\mathrm{CdTe}$ ). Focusing on CdTe, this type of QD can be made to be water-soluble, so it can be applied to the prints in an aqueous solution. It can also it can be made to fluoresce at different colors based on the size of the particle. As a result, it can be an effective detecting agent on surfaces of all different colors. After be ingrained with the water-soluble CdTe for a number of minutes, the prints produced are of far higher resolution than anything that can be produced by conventional methods.

Similarly to the gold nanoparticles, these QDs can also be functionalized with a variety of chemicals. In a newer study, CdTe QDs were capped with mercaptosuccinic acid (MSA) and this resulted in a much shorter time for the image to develop. Formerly, using CdTe QDs would require approximately 15 minutes of bathing in solution to produce a visible product, but the MSA capped CdTe QDS was able to produce visible prints after a mere second with a greater resolution than the best chemical print developers currently used.

Nano technology method: The technique makes use of the facts that antibody Nano particle conjugates can be synthesized to target amino acids which are present in fingerprints. Amino acids in sweat on non-porous surfaces are targeted by ANTI COTININE antibodies that bind to amino acids when the antibodies bound to the amino acids the Nanoparticles resolve the molecular compounds leading to a much clearer fingerprints picture. Antibodies are purpose built devises with distinct functional domains. Native antibodies are comprised of four polypeptide chains two heavy chains and two light chains joined by interchanging disulphide linkage.

Traditional gold solution is replaced with more stable equivalent wherein the gold Nanoparticles bristle with long hydrocarbon chains and are suspended in petrol ether.

They stick to the fingerprints residues through hydrophobic interactions and can be developed with silver producing high quality prints after just three minutes immersion time. but gold being unsuitable, it is stabilized by $\mathrm{n}$ alkanethiols. Enantio selective ANTI L AMIMO ACID antibodies conjugated to gold Nana particles are found to facilitate the detection of latent fingerprints by interacting with amino acids present in friction ridges secretions .this antibodies based and dried fingerprints on non-porous surfaces.

Iodine and a naphtoflavon method for skin: Finger print on skin can be detected by the use of iodine and a naphthalene iodine reacts with greases and oils left on surfaces from fingerprint iodine fumes are generated using a standard iodine fuming pipe and directed on to skin through a funnel, approximately 30 seconds exposure provides sufficient iodine fuming to see the brownish outline of the fingerprint. The iodine also reacts with the background skin but allowing it to evaporate for several seconds improves the contrast. The naphthoflavone is dissolved in a chloroform cyclo hexane solution and is applied to the area in the foam of an aerosol spray application when the naphthoflavane reacts with the iodine a dark blue color chemical is produced which is easily seen against the skin.

\section{Multi metal deposition}

Currently, there are a multitude of techniques which police utilize in detecting latent fingerprints, including optical methods, physical methods (such as dusting), and Chemical methods: While these methods are effective, there is still much room for improvement. The first technique that uses nanoparticles for fingerprint detection is called multi-metal deposition (MMD) and involves a two-step chemical process. First, an object would be placed in an acid bath containing gold nanoparticles, in which the gold attaches itself to the ridges of the finger print left on the object. Next, the object is soaked in a bath of silver nanoparticles, where the gold layer acts as a nucleation site for the silver to build onto, thus forming a visible print. This technique can be augmented by the addition of functional groups onto the gold particles. One interesting example was created in which a specific antibody that is related to nicotine is attached onto the gold nanoparticles. During experimentation, the solution could not only effectively reveal fingerprints, but the fingerprints themselves would also fluoresce under UV light if the donator of the prints was a smoker. While this study focused specifically on smoking, there is no reason to think that this technique could not be adapted to incorporate other indicators for a variety of drugs or other sweat-secreted chemicals.

MMD is a highly versatile technique because it has been shown to be effective on both porous and non-porous surfaces as well as surfaces that are wet. However, it is limited by the fact that it requires the object with the fingerprints on it to be bathed in an aqueous solution of the gold nanoparticles. It is thus not useful for finding prints on surfaces such as walls or floors at the scene of the crime or for any object too large to be soaked in a desk top bath. Another technique that scientists are testing involves improving the conventional method of applying a fine dust to the site and then brushing away excess, leaving only the particles that adhere to the ridges of the print.

Scientists are now experimenting with dust that has nano- 
scale particles rather than the classical micron-range sized particles. These studies have shown that when gold is used as the metal for the nanoparticles, nano-dusting has been able to produce higher resolution prints on a variety of surfaces. Having high resolution prints is important because the detail of a print is very fine, and slight imperfections can dramatically affect the ability of the police to use them in an investigation. This is especially true in the case of partial prints, when only a portion of the fingerprint is found. Tests with other types of nanoparticles such as zinc oxide and iron oxide have also been successful at producing high resolution prints through dusting. The zinc oxide was shown to be able to effectively lift prints on non-porous surfaces even when the prints have been aged for longer than a month. Tests have also been conducted on titanium dioxide particles and have revealed that it generally has the same effectiveness as conventional dusting methods. Interestingly, this method is very good for lifting prints off of the sticky side of tape when dissolved in a solution of methanol.

\section{Laser method of fingerprint development}

Laser an acronym for light amplified stimulated emission of radiation has become in valuable tool in the hands of forensic scientist. They are extensively used in forensic examination of fibers, paint, documents, fractured glass etc. Laser is now used for developing of latent fingerprints too. Some of state finger print bureaus like Haryana, Tamil Nadu and New Delhi using laser equipment.

If inherent luminance fails to detect latent fingerprints through laser then the same can be treated with fluorescent material like COUMARIN - 6 to give induced luminescence to latent fingerprints very old prints even as old as 10 years can be developed using laser method on surfaces like plastic, rubber, painted walls wood, lather.

The main advantage of laser beam over the conventional fingerprints development methods is its capability to develop latent prints that cannot be developed by any other method. Id it is highly sensitive method and does not suffer from any time limitation. The developed fingerprints show off markedly under laser source. This method is highly evolved and can become the method of choice to be followed by other conventional methods. Through the equipment is expensive it is best nondestructive method.

\section{Conclusion}

Difference between conventional methods and modern methods for developing fingerprints In conventional methods there is some draw backs suppose if we talk about powder methods there is problem how to select different color powders according to surface, if we take modern methods like quantum dots method we can use any surfaces to develop fingerprints there is no selection of powders according to surface. in physical developer it take more time to develop if we take Nano technology method clear print can develop by three minutes, conventional method like carbon black method

\begin{tabular}{|c|c|}
\hline \multicolumn{2}{|l|}{ Observation Table } \\
\hline $\begin{array}{c}\text { Conventional methods for examination of } \\
\text { fingerprints }\end{array}$ & $\begin{array}{l}\text { Modern methods for Examination } \\
\text { fingerprints }\end{array}$ \\
\hline $\begin{array}{l}\text { Powder methods: For dark surfaces } \\
\text { grey or white powder use for light } \\
\text { surfaces black powder will use main } \\
\text { problem to select suitable powder to use. }\end{array}$ & $\begin{array}{l}\text { Quantum Dots(Q.D): Q.D can be an } \\
\text { effective detecting agent on surfaces } \\
\text { all different colors. Prints after mere } \\
\text { seconds with great resolution than the } \\
\text { best chemical print developer currently } \\
\text { used. }\end{array}$ \\
\hline $\begin{array}{l}\text { Physical developer: Used to detect } \\
\text { dark grey black surfaces on porous. It } \\
\text { takes more time and expensive. }\end{array}$ & $\begin{array}{l}\text { Nano technology: They stick to } \\
\text { fingerprints residues through hydrophobic } \\
\text { and develop silver producing high quality } \\
\text { prints with Three Minutes. Can Examine } \\
\text { Old And Dry Prints }\end{array}$ \\
\hline $\begin{array}{l}\text { lodine fuming: Natural oils and body } \\
\text { fats absorb iodine. It can use paper card } \\
\text { board not in metallic surfaces. }\end{array}$ & $\begin{array}{l}\text { lodine and naphto f lavone methods } \\
\text { for skin: When iodine reacts with } \\
\text { naphtoflavane gives dark blue. Easily } \\
\text { Can Seen Against Skin. }\end{array}$ \\
\hline $\begin{array}{c}\text { Carbon black: Made with binder, } \\
\text { charcoal the main disadvantage Create } \\
\text { Mess And Cannot Work On Porous } \\
\text { Medium }\end{array}$ & $\begin{array}{l}\text { Multi metal deposition: It can use } \\
\text { both porous and non-porous surfaces } \\
\text { examination. } \\
\text { Gold nano particles useful to finding } \\
\text { prints on surfaces like walls and floors in } \\
\text { crime scene. }\end{array}$ \\
\hline $\begin{array}{l}\text { Ninhydrin method: Development needs } \\
\text { long time and storage is difficult. } \\
\text { VMD: difficult to develop fingerprints on } \\
\text { heavily plastic polymer. }\end{array}$ & $\begin{array}{l}\text { Laser: Very old } 10 \text { years can detect } \\
\text { plastic, rubber, painted walls, paper } \\
\text { wood etc.. } \\
\text { Main Advantage Develops Latent Prints } \\
\text { That Cannot be Developed By Any Other } \\
\text { Method. }\end{array}$ \\
\hline
\end{tabular}

it will create mess on developed fingerprint it is difficult to see developed latent fingerprints.

Nano technology methods Highly used for porous, nonporous and wet surfaces by using Nano technology method we can examine latent fingerprints on different surfaces if we take modern method like multi metal deposition method the advantage of multi metal deposition method is it can analyses the fingerprint donor is smoker or not, and multi metal deposition method have capable to analyze the fingerprint donor is drug addict or not, suppose if we take conventional method VMD it cannot analyses latent finger prints on plastic polymer if we take modern method.

LASER it can analyze 10 years old prints also and can examine fingerprints on plastic, rubber, pain walls leather etc. The main advantage of LASER method is it will develop latent fingerprints very accurate manner no conventional method cannot develop latent fingerprints like LASER method .Day by day technology was increasing and changing, and crimes also increasing, for examination of latent fingerprints modern methods are best to get latent fingerprints very accurate manner with in less time .But new methods also having drawbacks, but compare to conventional method modern methods is best in all the ways.

\section{References}

1. MSS Indira Sudha. Biometrics and fingerprint.

2. Nabar BS. Forensic science in crime investigation.

3. France J. Nano Technology and Latent Fingerprint Detection. da vinci's Notebook. 20014; 6.

4. https://www.slideshare.net>faraharooj 